\title{
Analysis of E-commerce Business Strategy - Take JD.com, Inc. as the Case
}

\author{
Zilin Huo ${ }^{1} \&$ Jiyuan $\mathrm{Du}^{2}$ \\ ${ }^{1}$ School of Business, Suzhou University of Science and Technology, Suzhou, Jiangsu, China \\ ${ }^{2}$ Faculty of Artificial Intelligence, Nanjing Vocational College of Information Technology, Nanjing, Jiangsu, \\ China \\ Correspondence: Zilin Huo, School of Business, Suzhou University of Science and Technology, Suzhou, Jiangsu, \\ China. E-mail: zilin.huo@outlook.com
}

Received: January 5, 2022

doi:10.20849/abr.v7i1.989
Accepted: January 25, 2022

Online Published: January 26, 2022

URL: https://doi.org/10.20849/abr.v7i1.989

\begin{abstract}
The development of e-commerce has brought a lot of convenience to people. People can shop without going out with computers or mobile phones, and the goods are cheaper. With the development of e-commerce, e-commerce has become an important part of people's daily life. And JD.com, Inc, as a leading supply chain based technology and service enterprise, has built its own nationwide fulfillment infrastructure and last-mile delivery network. According to the Fortune Global 500 Companies by revenue in 2021, JD has been the largest retailer in China. At the same time of doing its retailing, with its advantages in customer services and operational efficiency and its strategy of long-term investment in services and logistics industry, JD has established a huge scale advantage and owned a market leading position. This article is focused on the development status and trend analysis of e-commerce in parallel with the market performance of JD.
\end{abstract}

Keywords: e-commerce, business strategy, competitive analysis, marketing, supply chain

\section{Industry Analysis}

In recent years, with the rise of JD.com, Fanke, Suning, Tesco, Gome, etc., B2B models have become more and more popular. Now almost all media have established B2C platforms, and even Thunder, Tianya, Baidu, etc. have established B2B malls. With the prevalence of B2B, people are beginning to think that the era of B2C is coming, and does this herald that more and more consumers will use the Internet to directly participate in economic activities? B2C business model is that merchants search for suitable consumer groups through the Internet to truly realize customized consumption. For consumers, it not only reduces the cost, but also shortens the transaction time, which is an ideal consumption model. For merchants, they can receive feedback from consumers as soon as possible, which is conducive to merchants constantly changing their sales strategies while winning more revenue.

\section{Introduction of JD.com, Inc}

JD.com, Inc is China's second-largest e-commerce company after Alibaba in terms of transaction volume, offering a wide selection of authentic products at competitive prices, with speedy and reliable delivery. The company has built its own nationwide fulfilment infrastructure and last-mile delivery network, staffed by its own employees, which supports both its online direct sales, its online marketplace and omnichannel businesses.

\section{Comprehensive Analysis}

There is a very friendly policy and entrepreneurship environment in China. High-tech companies are supported priority due to the dramatically development and raising requirement of online shopping and domestic advanced logistics. At this stage, a large amount of middle high school students trend to learn computer science and technology, so JD seizes the opportunity to hire lots of college graduates with a degree of science in a competitive lower cost. For other hand, JD's CEO Mr. Liu Qiangdong designed JD Group's formula through registered in Cayman Islands. Those are the reasons of why it has been developed in a such speed and successfully listed on Nasdaq and HKEX via VIE structure.

Nowadays, China is gradually coming out of the influence of COVID-19. This is a very exciting site that JD has been expected so that its business partners also can recover quickly from the pandemic, relying on the online and 
offline supply chain infrastructure of JD.

As it vividly shows in JD's Financial and Operational Highlights announced on its official page, it's no doubt that JD has made the remarkable progress from 2012 to 2020. JD are developing to be the largest e-commerce company in China and it will share a percent of 15.1 until the year 2023.

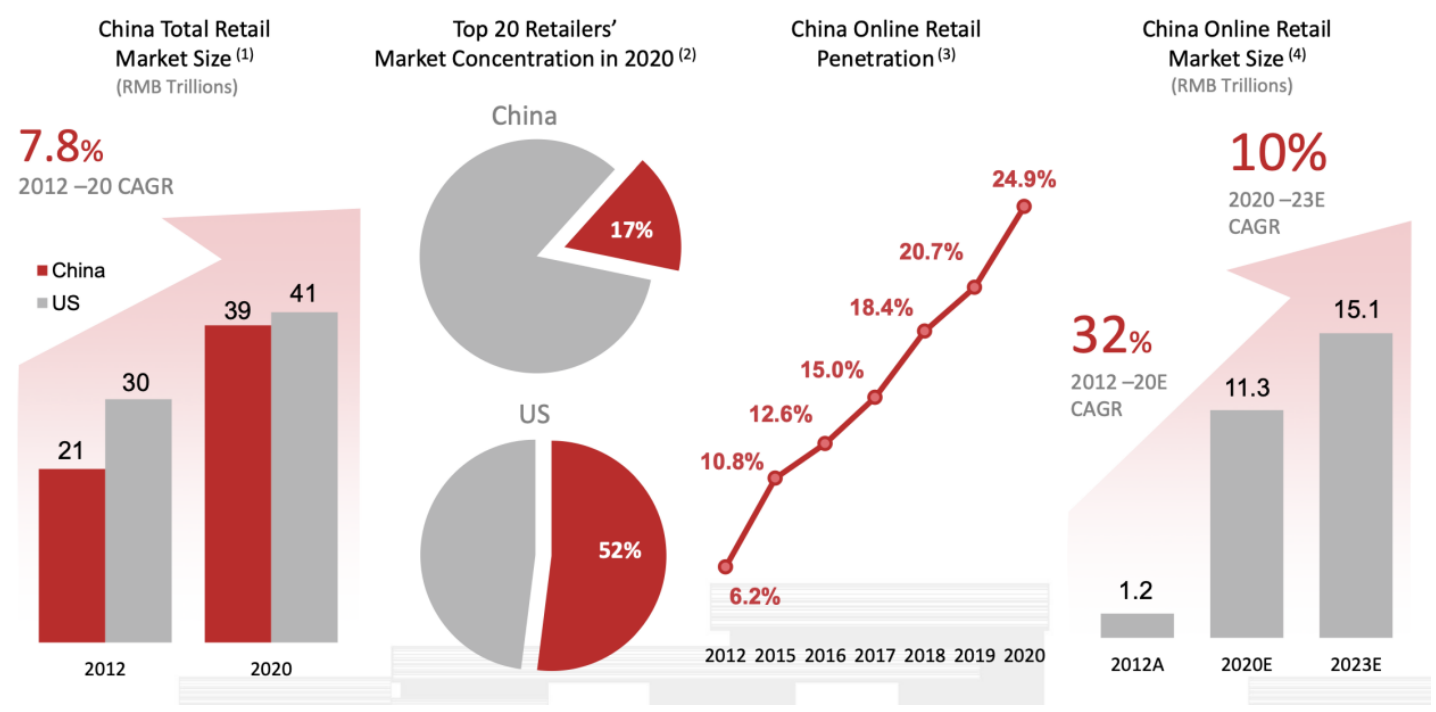

Figure 1. Financial and Operational Highlights of JD.com, Inc

Though changes in China's or global economic, political or social conditions or government policies may have a material and adverse effect on JD's business and operations, JD still be confronted with every threats it faced. If Chinese government deems that the contractual arrangements in relation to our variable interest entities do not comply with its regulatory restrictions on foreign investment in the relevant industries, or if these regulations or the interpretation of existing regulations change in the future, JD could be subject to severe penalties or be forced to relinquish its interests in those operations.

JD has completed listing and financing, and has abundant internal capital development. It has invested a lot of money in the quality of goods, so as to ensure the quality of each commodity and make consumers feel satisfied. So far, only JD has its own logistics system, which greatly improves the delivery timeliness. JD has also recently adopted door-to-door service, which is favored by the majority of people in China. The following picture shows the provinces covered by JD Logistics: 

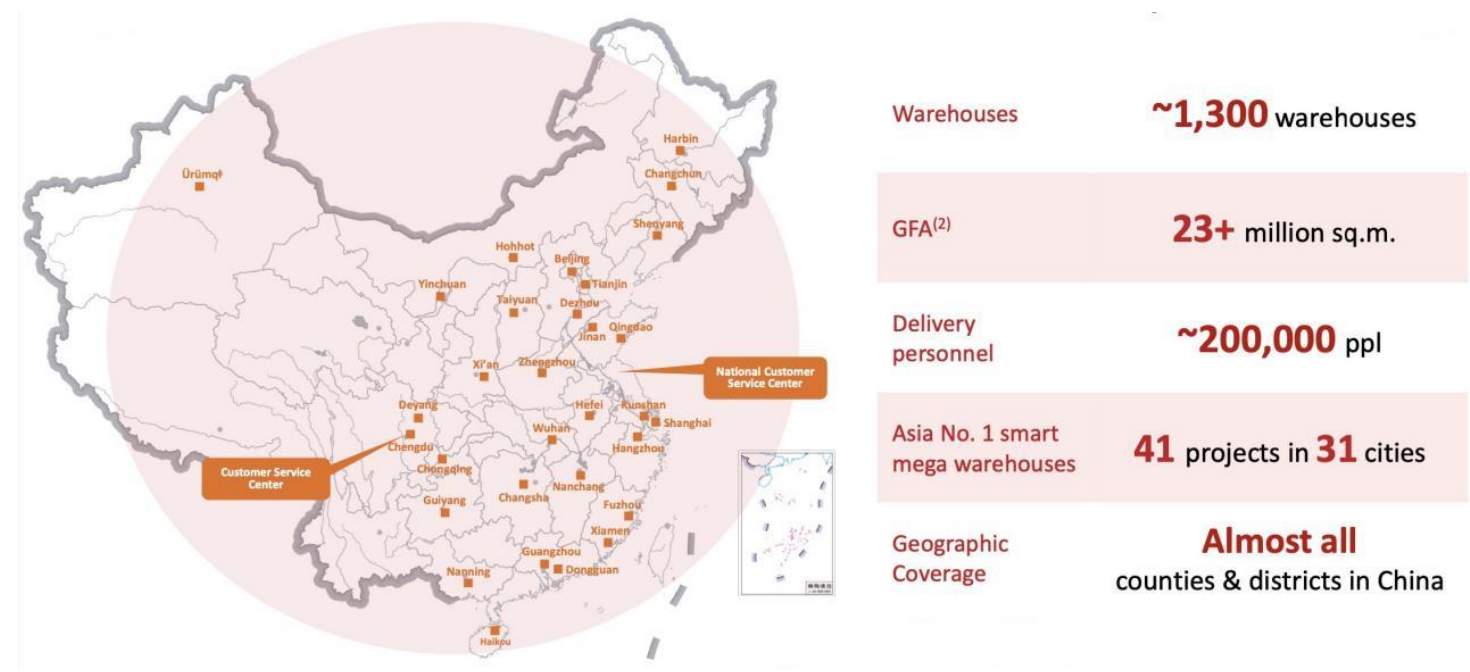

Figure 2. The Scale of JD Logistics in China

From this picture we can see that JD Logistics covers many provinces. JD Logistics has designed and developed an extensive and integrated logistics network to reduce intermediate links and shorten performance time. Its integrated supply chain technology can intelligently place inventory in the warehouse closest to potential end consumers. When customers place orders, JD Logistics can deliver goods from the warehouse nearest to end consumers, so as to achieve rapid performance and obtain high consumer satisfaction. And JD Logistics applies technology to all key links of the supply chain and has built a comprehensive intelligent logistics system to realize service automation, operation digitization and intelligent decision-making.

\section{Competitive Analysis}

However, compared with other enterprises, JD started relatively late and many of its implementation efficiency is not such reasonable. JD's logistics can be seen all over China and many goods are delivered directly to the doors, which would consumes a lot of human and material resources, which is a great challenge for JD.

Faced with those inside weaknesses and outside threats, the opportunities are open to JD if handled correctly. Nowadays, the increasingly developed network has brought great advantages to many enterprises, promoted the development of relevant industries and extremely improved the operation rate of logistics. At the same time, JD is also the beneficiary of the rapid development of network. JD is gradually developing scientific and technological intelligent logistics. If a large logistics enterprise like JD has logistics quality assurance, it will It has brought great benefits to the enterprise and the company's operating efficiency. JD's offline stores are increasing quickly. If the goods are broken or have problems, JD's customer service can provide online dispute resolution, offline goods return and exchange, customer evaluation, etc. It can be seen that the development of science and technology has brought great operating efficiency to JD and many opportunities for enterprises.

But many enterprises also pose JD with great competitive pressure. Well-known brands such as PDD and Alibaba are JD's main competitors. In particular, many people are pouring out of many live platforms, which also brings great stresses to JD.

\section{Strategy Suggestion}

JD's future strategy need to focuses on quality growth. In order to achieve its strategic objectives, JD should coordinate its market leadership in the field of e-commerce and improve the technical service capacity based on the supply chain to enable every link of the industrial chain. JD's team, organization and corporate culture are the cornerstone of its successful growth strategy. They have to further expand the scale and enhance economies of scale, further enhance customer experience by enhancing user interaction and expanding customer base Improve the technical service capability based on supply chain, and strengthen its team, organization and JD's corporate culture.

\section{Summary \& Future Anticipate}

In the future, the trend of cross-border mergers and acquisitions in China's e-commerce service industry is obvious. The development of online digital services will enter the fast track, and e-commerce services will promote the 
development of flexible supply chains. At the same time, thanks to the improvement of national living standards, more and more people tend to go shopping on overseas and it helps to improve their quality of life. Cross border e-commerce has sprung up against this background. JD.com, tmall.com and other large e-commerce platforms have launched their own overseas outsourcing channels. It's worth to looking forward that overseas shopping will be more and more convenient and faster!

\section{References}

Edutt.com. (2021, July 25). Analysis on the Development Status and Trend of E-commerce. Retrieved December 31, 2021, from https://m.edutt.com/news_show_1872376/

Fengqu de Xiaofang. (2021, September 25). SWOT Analysis of JD's Market Strategy. Retrieved December 29, 2021, from https://www.zhihu.com/column/p/413883427

HKEX. (2021). 2020 Annual Report of JD.com, Inc. Retrieved December 29, 2021, from https://www1.hkexnews.hk/listedco/listconews/sehk/2021/0418/2021041800020_c.pdf

JD.com, Inc. (2021). Annual Report Pursuant to Section 13 or 15(d) of the Securities Exchange Act of 1934 for the fiscal year ended December 31, 2020. Retrieved December 29, 2021, from https://ir.jd.com/static-files/e5d705b1-3089-4247-8b12-a4fb69fc025a

JD.com, Inc. (2021). Financial and Operational Highlights. Retrieved December 29, 2021, from https://ir.jd.com/system/files-encrypted/nasdaq_kms/assets/2021/11/18/4-46-48/JD.com\%20Inc\%203Q2021 \%20Financial\%20and\%20Operational\%20Highlights.pdf

Nasdaq. (2021). Introduction of JD.com, Inc. Retrieved December 29, 2021, from https://www.nasdaq.com/market-activity/stocks/jd

\section{Copyrights}

Copyright for this article is retained by the author(s), with first publication rights granted to the journal.

This is an open-access article distributed under the terms and conditions of the Creative Commons Attribution license (http://creativecommons.org/licenses/by/4.0/). 